\title{
PATENTES EM ENERGIAS RENOVÁVEIS: UM ESTUDO EXPLORATÓRIO A PARTIR DE INDICADORES PARA PAÍSES SELECIONADOS ${ }^{\wedge}$
}

\author{
RENEWABLE ENERGIES PATENTS: AN EXPLORATORY STUDY \\ FROM INDICATORS FOR SELECTED COUNTRIES \\ Dienifer Regina Fortes Storti* \\ Orlando Martinelli Junior**
}

enviado: 10 noviembre 2020 - aceptado: 11 mayo 2021

\begin{abstract}
Resumo
Este artigo quantifica e analisa os esforços tecnológicos, os perfis de especialização e os movimentos tecnológicos em energias renováveis dos vinte principais países consumidores de energia no período de 1992 a 2018. Utiliza-se uma base de patentes em treze áreas de energias renováveis para a construção de indicadores estruturais de inovação, índices de vantagem tecnológica revelada e aplicação do método shift-share. Os resultados mostraram que os Estados Unidos estiveram à frente da maioria dessas áreas, entretanto, os esforços inovativos mais dinâmicos foram os do Japão, Coreia do Sul e China. A análise shift-share indicou que os esforços tecnológicos dos países não focam em uma fonte energética alternativa, mas conectam-se a um leque mais amplo de tecnologias em energias renováveis.
\end{abstract}

Palavras-chave: energias renováveis, patentes, ecoinovações, economia da inovação. Classificação JEL: Q42, Q55.

O presente trabalho foi realizado com apoio da Coordenação de Aperfeiçoamento de Pessoal de Nível Superior - Brasil (CAPES) - Código de Financiamento 001. Fortes Storti, D. R., \& Martinelli Junior, O. (2022). Patentes em energias renováveis: Um estudo exploratório a partir de indicadores para países selecionados, Estudios económicos, 39(78), pp. 127-156. DOI: https://doi.org/10.52292/j. estudecon.2022.2267

* Universidade Federal de Santa Maria e Universidade Estadual de Campinas, Brazil. ORCID: https:// orcid.org/ 0000-0003-4705-8309. E-mail: dieniferstorti@gmail.com

** Universidade Federal de Santa Maria, Brazil. ORCID: https://orcid.org/ 0000-0002-7548-8464. E-mail: orlando.martinelli@gmail.com 


\begin{abstract}
This article quantifies and analyzes technological efforts, specialization profiles, and technological movements in renewable energy in the twenty main energyconsuming countries, from 1992 to 2018. A database patent is used in thirteen areas of renewable energy for the construction of structural innovation indicators, of revealed technological advantage indexes and for the application of the shift-share method. The results showed that the United States was ahead of most patents areas in renewable energy, however, Japan, South Korea and China have been revealed more dynamic countries on innovative efforts. The shift-share analysis indicated that the countries' technological efforts do not focus only on one alternative energy source, but they connect to a wider range of renewable energy technologies.
\end{abstract}

Keywords: renewable energy, patents, eco-innovations, innovation economics. JEL Codes: Q42. Q55. 


\section{INTRODUÇÃO}

Os possíveis limites do esgotamento das reservas fósseis, as recorrentes crises mundiais no setor petrolífero - que causam instabilidades e incertezas econômicas e geopolíticas - e as crescentes externalidades negativas socioambientais, derivadas do excesso de utilização de energias fósseis, estão induzindo, em diversos países, a criação de incentivos para a produção e o uso crescente de energias renováveis (daqui para adiante, ER). Especialmente a partir da Conferência Rio-92 e, posteriormente, com os acordos assumidos no Protocolo de Kyoto, são criados compromissos políticos e institucionais mais robustos para a implementação de diretrizes internacionais alinhadas com a preservação ambiental, incluindo mudanças no padrão de consumo, processos mais eficientes na produção de energia e incentivos de tecnologias em energias renováveis. Com isso, ganham corpo vetores econômicos, sociais e institucionais que estimulam um processo de construção de uma matriz energética baseada em energias renováveis (Renewable Energy Policy Network for the 21st Century [REN21], 2019).

As marcantes diferenças existentes nos aspectos geográficos e técnico-econômicos entre as formas de produção, comercialização e uso de energias renováveis, comparativamente às energias fósseis, fazem, porém, com que esse processo de transição não seja neutro para agentes econômicos (empresas e consumidores), setores produtivos nem para as economias. No plano econômico, implica a aceleração da taxa de depreciação dos investimentos realizados em energias tradicionais e a reorganização dos mercados (que podem mudar de oligopolizados para mercados mais competitivos e descentralizados, ou mesmo a criação de novos oligopólios configurados por agentes emergidos com novos mercados de novas fontes de energias). No plano do poder político e geoestratégico, a mudança energética redimensiona o papel de certos atores no plano internacional, em função da menor dependência do petróleo importado de poucas regiões produtoras (Criekemans, 2018). Nessa perspectiva, as potencialidades de exploração econômica das tecnologias em energias renováveis, para além das temáticas ambientais e do desenvolvimento sustentável, também se conectam com as das relações internacionais e da competição geoeconômica entre países - influenciando, inclusive, as negociações internacionais sobre o tema ambiental como um todo (Bonnet, Hache, Seck, Simoën \& Carcanague, 2019).

A partir desse pano de fundo, o presente artigo tem como principal objetivo quantificar e analisar os esforços tecnológicos, os perfis de especialização e os movimentos tecnológicos em energias renováveis dos vinte principais países consumidores de energia no período de 1992 a 2018. Metodologicamente, utiliza-se 
uma base de patentes em treze áreas de energias renováveis, para a construção e análise de indicadores estruturais de inovação, de índices de vantagem tecnológica revelada (VTR) e para a aplicação do método shift-share para esses países.

O artigo está organizado em quatro seções, além desta introdução. A seguir são apresentados elementos da vertente teórica neo-schumpeteriana, especialmente resgatando o conceito de regimes tecnológicos, que buscam amparar o entendimento sobre as características e complexidades dos processos de inovação, bem como situações de trajetórias de especialização tecnológica (ou não) dos países. $\mathrm{Na}$ segunda seção é apresentado um panorama da oferta e dos investimentos em ER. Na terceira, tem-se a parte metodológica, contendo a descrição e a fonte dos dados, a construção, o cálculo e os resultados dos indicadores, índices, e da análise shift-share. A quarta seção apresenta as conclusões do trabalho.

\section{REGIMES TECNOLÓGICOS, INOVAÇÃO, E ESPECIALIZAÇÃO TECNOLÓGICA}

Este artigo se ampara no conceito de regimes tecnológicos (RT), que reúne tanto os elementos e/ou atributos de uma determinada tecnologia (stricto sensu), bem como outras características sistêmicas, que determinam suas potencialidades (técnicas, econômicas e sociais) para a sua difusão. Os RTs podem ser analisados a partir de quatro dimensões: oportunidade, apropriabilidade, cumulatividade e a base de conhecimento (Malerba \& Orsenigo, 1993) ${ }^{1}$. Especialmente no caso de inovações associadas a trajetórias tecnológicas novas, os estímulos de oferta (supply push) e de demanda (demand pull) podem ser importantes para modificar os RTs e, assim, impactar o processo de difusão e adoção. Estímulos de oferta podem ser implementados por diversos tipos de políticas públicas (desde os econômicos, financeiros e legais, até os de formação educacional) que reduzem as barreiras e/ou os custos e/ou as incertezas para o desenvolvimento de tecnologias novas. Políticas públicas também podem estimular a demanda, através de medidas que diminuam o custo de adoção (e.g. redução de taxas, implementação de padronização técnica

1 A oportunidade tecnológica é a possibilidade de aplicação ou uso comercial da inovação e do potencial de difusão da inovação em termos de produtos e de mercados. A apropriabilidade diz respeito às possibilidades e/ou formas de proteger as inovações frente às imitações. A cumulatividade do conhecimento associa-se às características da natureza cognitiva e dos processos de aprendizagem relacionados à tecnologia e de sua aplicação no processo inovativo. A base do conhecimento relaciona-se especialmente ao grau de complexidade e ao domínio do conhecimento científico para se inovar (Malerba \& Orsenigo, 1993). 
etc.) e/ou que acelerem a adoção da nova tecnologia - em ambos os movimentos o mercado é ampliado, podendo desencadear a geração de economias de escala e de escopo, elevando a rentabilidade esperada (Samant, Pooja \& Hatfeld, 2020).

A partir de Schumpeter, a literatura tem desenvolvido duas visões sobre especialização tecnológica ou a sua disrupção: situações de "destruição criativa" e de "acumulação criativa". A diferença entre as duas depende de como se configuram as propriedades dos regimes tecnológicos e do processo inovativo nos setores produtivos.

A visão de "destruição criativa" aborda a especialização associada a um regime tecnológico irregular e aleatório. Diferentemente, a visão de "acúmulo criativo" trata a especialização como resultado da acumulação interna de competências tecnológicas construídas no passado. Os componentes tácitos e específicos podem gerar importantes barreiras à entrada em mercados (Cefis, 2013; Feitosa, 2016).

Para os propósitos deste artigo, as inovações em energias renováveis - induzidas por fatores econômicos, institucionais e/ou socioambientais - podem ser relacionadas às duas visões. Considerando a visão do "acúmulo criativo", as inovações ocorrem de forma mais regular ao longo de trajetórias tecnológicas estabelecidas, e as empresas realizam inovações em outras fontes de energias renováveis como rotina para evitar a entrada de novos concorrentes. Já na visão da "destruição criativa", as novas tecnologias em energias renováveis promovem transformações nas atividades produtivas, permitindo a entrada de novas firmas, que buscam a exploração de oportunidades emergentes. Consequentemente, os países são impulsionados a maiores transformações nos seus campos de especialização tecnológica (Feitosa, 2016).

\section{ENERGIAS RENOVÁVEIS: UM BREVE PANORAMA}

Energia renovável é aquela que vem de fontes naturalmente reabastecidas e capazes de serem disponíveis na natureza por um longo tempo e/ou se regenerarem permanentemente. Exemplos de tais fontes podem ser o sol (energia solar), os mecanismos naturais do ambiente (como energia geotérmica, movimento das marés, movimento humano), a biomassa (energia fotossintética). As tecnologias aplicadas a essas fontes permitem transformá-las em formas utilizáveis de energia - especialmente, eletricidade, calor e combustíveis. A produção e utilização de energias renováveis ainda são minoritários na matriz energética mundial. Em 2018, cerca de $80 \%$ dessa matriz era de fontes fósseis, sendo $36 \%$ do petróleo, $13.2 \%$ do 
carvão e 31\% do gás natural. A energia nuclear e a energia renovável representaram $11 \%$ e $8 \%$, respectivamente (Yuehong et al., 2020). No entanto, a parcela relativa das energias renováveis no total cresceu de $5.9 \%$ em 2009 para 13.4\% em 2019 (Frankfurt School-UNEP Centre/BNEF, 2020).

Considerando a classificação da International Renewable Energy Agency (IRENA, s.f.), tem-se que a energia hidroelétrica é o tipo de energia renovável mais importante para a geração de eletricidade, seguida da nuclear. No entanto, o crescimento das energias eólica e solar ganham destaque (Tabela 1).

Tabela 1. Geração de Eletricidade por tipo de Energia

$$
\text { Renovável - GWh 2000-2018 }
$$

\begin{tabular}{lcccccc}
\hline \multicolumn{1}{c}{ Tipo } & 2000 & 2006 & 2012 & 2015 & 2018 & $\begin{array}{c}\text { Variação } \\
(\%)\end{array}$ \\
\hline BioC & 113780 & 181514 & 324305 & 415631 & 518467 & 355.7 \\
\hline Resíduos & 49544 & 61242 & 97387 & 101843 & 118773 & 139.7 \\
\hline Nuclear & 2590624 & 2791471 & 2460285 & 2570070 & 2710430 & 4.6 \\
HidroE & 2695591 & 3124333 & 3759303 & 3982151 & 4325111 & 60.5 \\
\hline GeoT & 52171 & 59611 & 70275 & 80562 & 88956 & 70.5 \\
\hline Solar & 1326 & 5885 & 103588 & 259681 & 565703 & 42562.4 \\
\hline Eólica & 31348 & 133668 & 525594 & 833732 & 1273409 & 3962.2 \\
\hline Marítima & 0546 & 0490 & 0496 & 1006 & 1005 & 84.1 \\
\hline Outras & 22049 & 29670 & 33734 & 35741 & 34662 & 57.2 \\
\hline Total & 5556979 & 6387884 & 7374967 & 8280417 & 9636516 & 73.4 \\
\hline
\end{tabular}

BioC $=$ Biocombustíveis; HidroE $=$ Hidroenergia GeoT $=$ geotérmica

Fonte: elaboração dos autores. Dados básicos : IEA - (International Energy Agency) - https://www.iea. org/data-and-statistics/data-tables 
Esses últimos dois tipos de energia são os que estão atraindo os maiores investimentos. Entre 2009 e 2019, dos US\$ 2.8 trilhões investidos, a parcela da solar foi $52 \%$, e a da eólica, $41 \%$. Em 2019, o valor investido correspondeu a cerca de três vezes o valor investido em carvão, gás natural e nuclear combinados (Frankfurt School-UNEP Centre/BNEF; Global Trends in Renewable Energy Investment, 2020). Considerando os principais investidores, ganham destaque os países desenvolvidos, o crescimento dos investimentos da Índia e, especialmente, da China (Figura 1).

Figura 1. Investimento Global em Energias Renováveis (excluídos os investimentos em hidroenergia acima de 50MW) 2009-2019

\section{• China Índia — Outros Países não Desenvolvidos — Países Desenvolvidos}

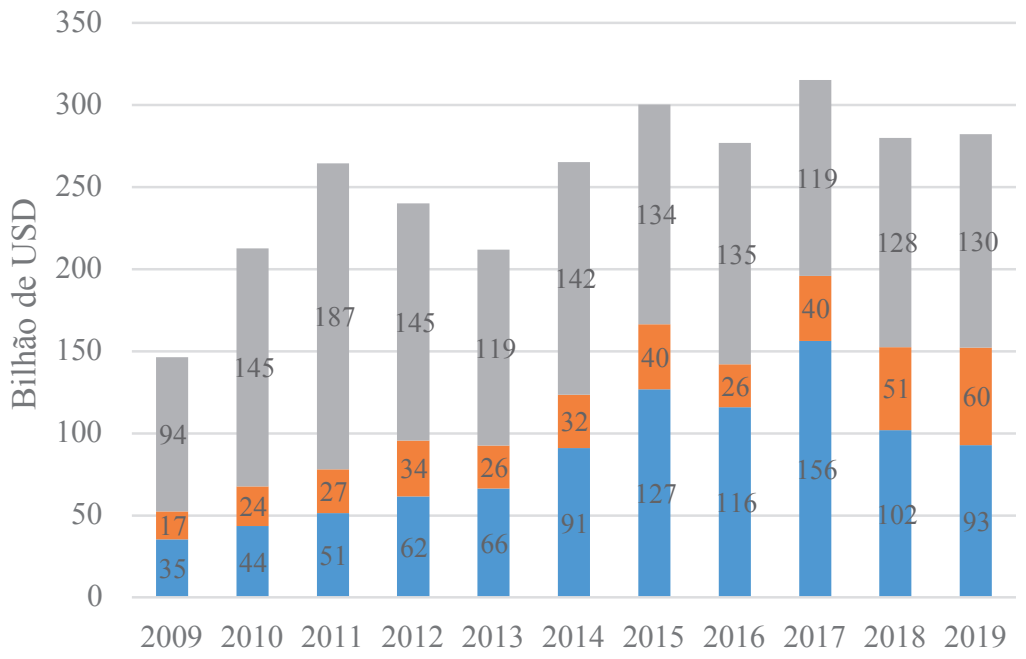

Fonte: Frankfurt School-UNEP Centre/BNEF.

Vale dizer que o grupo de países mais consumidores de energia e emissores de $\mathrm{CO}_{2}$ são os que estão investindo relativamente em ER. Diversos fatores têm impulsionado os investimentos em energia renovável. O principal deles são os acordos internacionais para a mitigação das mudanças climáticas e para a redução da poluição do ar. Tais acordos induzem - inclusive em função da crescente pressão 
política de diversos grupos sociais - a implementação de vários tipos de políticas públicas $^{2}$ no sentido de criar e difundir tecnologias em energias renováveis. No plano econômico, à medida em que se desenvolvem e difundem novas tecnologias, a redução de custos no tempo (via economia de escala, de escopo ou mesmo via subsídio) viabiliza economicamente a construção de novos mercados e modelos de negócio viáveis para os investimentos privados ${ }^{3}$.

\section{DESCRIÇÃO DOS DADOS E METODOLOGIA}

Este presente artigo utiliza as patentes em energias renováveis para a construção de indicadores de esforços tecnológicos, de perfis de especialização, e de desempenho tecnológico, bem como para a aplicação do método de decomposição estrutural (shift-share) aos principais países consumidores de energia. Uma vez que as patentes são disponibilizadas em diversas categorias, o seu uso permite mensurar não apenas as atividades inovativas, mas também analisar a direção que elas estão percorrendo, indicando quais setores estão apresentando maior progresso técnico ${ }^{4}$. O presente estudo não se propõe a realizar uma revisão bibliográfica extensa e minuciosa. Entretanto, alguns trabalhos podem ser citados de forma a fornecer uma breve visão sobre como o tema vem sendo trabalhado, contextualizando o atual estado da arte.

Os trabalhos de Feitosa (2016) e Bueno, Silveira, Buainain e Dal Poz (2018) utilizam dados de patentes, mas detêm objetivos e metodologias distintas. Enquanto o primeiro utiliza o cálculo de indicadores para caracterizar a estrutura tecnológica brasileira com base em classes de patentes, o segundo utiliza mineração de dados e construção de redes para clusters de fronteira tecnológica no setor de Bioenergia. Dentre os estudos que utilizam modelos econométricos, o trabalho de $\mathrm{Fu}, \mathrm{Li}$, Ondrich e Popp (2018) investiga a influência das políticas públicas no patenteamento de energia eólica nos 48 estados dos Estados Unidos, enquanto Bayer, Dolan e Urpelainen (2013) analisam um total de 74 países no período 1990-2009, a fim

2 Não é objetivo aqui detalhar tais políticas. Para maiores detalhes sobre isso, ver Storti (2020).

3 Um exemplo nesse aspecto: entre 2010 e 2019, o custo médio mundial de geração de eletricidade a partir de placas fotovoltaicas caiu de 0.37 centavos de dólar por kilowatt/hora para 0.07 centavos. A crescente automação industrial é considerada um dos fatores cruciais que explicam a queda nos custos de painéis fotovoltaicos. O custo médio de geração de eletricidade a partir de usinas hidroelétricas aumentou de 0.03 para 0.04 centavos de dólar, nesse período (Faria, 2020).

4 O uso de patentes em estudos de inovação também pode apresentar limitações (Archibugi e Pianta, 1996). No entanto, os estudos empíricos baseados em patentes, em geral, são os mais robustos devido à disponibilidade de dados em escala global e em diferentes níveis de análise (Albino et al., 2014) 
de identificar quais fatores econômicos e políticos exercem maiores influências nas inovações em energias renováveis.

III.1. Fonte e coleta de informação

O grupo de tecnologias renováveis foi delimitado pelas treze subclasses de patentes pertencentes à classe Produção de Energia Alternativa, que tem como base a classificação do IPC Green Inventory de patentes de tecnologias consideradas ambientalmente sustentáveis segundo as diretrizes estabelecidas pela Convenção Quadro das Nações Unidas sobre Mudanças Climáticas (UNFCCC, em inglês) 5 . Nessa classificação, as patentes podem tanto ser de tecnologias voltadas à geração de energia através de fontes renováveis, bem como de tecnologias que visam aumentar a eficiência de processos de geração e transmissão de energia, tais como redução de desperdícios e de danos ao meio-ambiente. O Quadro 1 apresenta as 13 subclasses, bem como os códigos correspondentes a cada uma delas.

Quadro 1. Subclasses de Produção de Energia Alternativa e seus respectivos códigos pela classificação IPC Green Inventory

\section{Subclasses de Produção \\ de Energia Alternativa}

\begin{tabular}{|c|c|}
\hline Biocombustíveis & $\begin{array}{l}\text { A01H; C02F 3/28; C02F 11/04; C07C 67/00; C07C 69/00; C10B } \\
\text { 53/02; C10G; C10L 1/00; C10L 1/02; C10L 1/14; C10L 1/19; C10L } \\
\text { 1/182; C10L 3/00; C10L 5/00; C10L 5/40 - 5/48; C10L 9/00; C11C } \\
\text { 3/10; C12M 1/107; C12N 1/13; C12N 1/15; C12N 1/21; C12N 5/10; } \\
\text { C12N 9/24; C12N 15/00; C12P 5/02; C12P 7/06- 7/14; C12P 7/64. }\end{array}$ \\
\hline IGCC & C10L 3/00; F02C 2/28. \\
\hline Células de combustível & $\begin{array}{l}\text { H01M 2/00 - 2/04; H01M 4/86 - 4/98; H01M 8/00 - 8/24; H01M } \\
12 / 00-12 / 08 \text {. }\end{array}$ \\
\hline $\begin{array}{c}\text { Pirólise ou gaseificação } \\
\text { de biomassa }\end{array}$ & C10B 53/00; C10J. \\
\hline
\end{tabular}

5 O chamado "International Patent Classification (IPC): Green Inventory" foi desenvolvido por um comitê de especialistas, com o objetivo de facilitar as buscas por informações de patente relacionadas às tecnologias ambientalmente saudáveis, a partir das determinações da Convenção Quadro das Nações Unidas sobre Mudanças Climáticas (em inglês: United Nations Framework Convention on Climate Change - UNFCCC). 
Aproveitamento de energia a partir de resíduos de atividades humanas
A62D 3/02; B01D 53/02; B01D 53/04; B01D 53/047; B01D 53/14; B01D 53/22; B01D 53/24; B09B 3/00; C02F 11/04; C02F 11/14; C10J 3/02; C10J 3/46; C10L 5/00; C10L 5/42; C10L 5/44; C10L 5/46 C10L 5/48; D21C 11/00; F23B 90/00; F23G 5/00; F23G 5/027; F23G 7/00; F23G 7/10.

\begin{tabular}{cl}
\hline \multirow{2}{*}{ Energia hidráulica } & B63H 19/02; B63H 19/04; E02B 9/00-9/06; F03B 13/12- 13/26; \\
& F03B 15/00- 15/22. \\
\hline OTEC & F03G 7/05. \\
\hline \multirow{2}{*}{ Energia eólica } & B60K 16/00; B60L 8/00; B63B 35/00; B63H 13/00; E04H 12/00; \\
& F03D 13/00; F03G 7/05. \\
\hline & B60K 16/00; B60L 8/00; C01B 33/02; C02F 1/14; C23C 14/14; \\
& C23C 16/24; E04D 13/00; E04D 13/18; C30B 29/06; F02C 1/05; \\
& F03D 1/04; F03D 9/00; F03D 13/20; F03G 6/00 - 6/06; F21L 4/00; \\
& F21S 9/03; F22B 1/00; F24D 3/00; F24D 5/00; F24D 11/00; F24D \\
& 17/00; F24D 19/00; F24S 10/10; F24S 23/00; F24S 90/00; F24V \\
& 30/00; F25B 27/00; F26B 3/00; F26B 3/28; G02B 7/183; H01G \\
& 09/20; H01L 25/00; H01L 25/03; H01L 25/16; H01L 25/18; H01L \\
27/30; H01L 27/142; H01L 31/00; H01L 31/078; H01L 31/042; & H01L 31/0525; H01L 51/42 - 51/48; H01M 14/00; H02J 7/35; H02S \\
10/00; H02S 40/44.
\end{tabular}

Energia geotérmica

H02N 10/00; F01K; F03G 4/00 - 4/06; F03G 7/04; F24F 5/00; F24T 10/00 - 50/00; F25B 30/06.

\begin{tabular}{cl}
\hline $\begin{array}{c}\text { Outros tipos de } \\
\text { produção ou utilização } \\
\text { de calor }\end{array}$ & F24D 11/02; F24D 15/04; F24D 17/02; F24H 4/00; F24T 10/00 \\
& $-50 / 00 ;$ F24V 30/00 - 50/00; F25B 30/00. \\
\hline & \\
$\begin{array}{c}\text { Utilização de calor } \\
\text { residual }\end{array}$ & C02F 1/16; C10J 3/86; D21F 5/20; F01K 17/00; F01K 23/04; F01K \\
& F01K 27/00; F01N 5/00; F02C 6/18; F02G 5/00-5/04; F03G 5/00 - \\
& 5/08; F22B 1/02; F23G 5/46; F24F 12/00; F25B 27/02; F27D 17/00; \\
& F28D 17/00-20/00. \\
\hline DPEEM & F03G 5/00-5/08.
\end{tabular}

IGCC $=$ Ciclo combinado de gaseificação integrada OTEC $=$ Conversão da energia térmica dos oceanos; DPEEM = Dispositivo para produção de energia mecânica a partir de energia muscular.

Obs: Os valores separados por traço indicam o intervalo de códigos que está sendo considerado.

Fonte: Elaboração própria com base no IPC Green Inventory. 
A coleta das patentes foi realizada na plataforma livre PATENTSCOPE do World Intellectual Property Organization (WIPO), entre 1992 e 2018, considerando dois períodos: o primeiro entre janeiro de 1992 e junho de 2005; o segundo entre julho de 2005 e dezembro de 2008. A coleta foi realizada para os vinte maiores países consumidores de energia no ano de 2015: China, Estados Unidos, Índia, Rússia, Japão, Brasil, Alemanha, Canadá, Irã, Indonésia, França, Coreia do Sul, Nigéria, Reino Unido, México, Arábia Saudita, Itália, Turquia, Austrália e Espanha, nessa ordem. Na área de ER, o número de patentes para esses países somou 46.952 no primeiro período, e 140.677 patentes no segundo. O ano 1992 foi escolhido como o inicial, pois se refere ao ano da Conferência Rio-92, que representou um marco no debate global sobre mudanças climáticas e, consequentemente, sobre uso de fontes de ER. O ano de 2005 foi a entrada em vigor do Protocolo de Kyoto, onde os países-membros (principalmente os desenvolvidos) firmaram compromissos com metas para reduzir a emissão de gases do efeito estufa.

\section{III.2. Indicadores estruturais de inovação}

A seguir são apresentados os indicadores utilizados para a análise das patentes em ER para os países e períodos temporais selecionados (Quadro 2). A construção empírica de indicadores sobre inovações tecnológicas é amparada pela contribuição importante de Soete (1987), ao utilizar tanto as patentes como proxy de força tecnológica, bem como o indicador de vantagem comparativa revelada para estimar um índice de vantagem tecnológica revelada (VTR) de países (Feitosa, 2016).

Quadro 2. Indicadores tecnológicos utilizados no estudo

\begin{tabular}{|c|c|}
\hline Indicador & Representação algébrica \\
\hline (1) Vantagem tecnológica revelada & $V T R_{i j}=\frac{P_{i j}}{\sum_{i} P_{i j}} / \frac{\sum_{j} P_{i j}}{\sum_{i} \sum_{j} P_{i j}}$ \\
\hline $\begin{array}{l}\text { (2) Taxa de variação da vantagem tecnológica } \\
\text { revelada }\end{array}$ & $\frac{\Delta V T R_{i j}}{V T R_{i j} \text { inicial }} * 100$ \\
\hline (3) Nível de oportunidade tecnológica & $O T_{i}=\frac{\sum_{j} P_{i j}}{\sum_{i} \sum_{j} P_{i j}}$ \\
\hline
\end{tabular}


(4) Índice de persistência $\quad I P=\frac{C_{i f}}{C_{t}}$

(5) Índice de mobilidade

$I M=1-I P$

(6) Coeficiente de correlação entre a $V T R_{i j}$ inicial e a variação da participação do país $\mathrm{j}$ no total de patentes mundiais na subclasse i.

$$
\begin{aligned}
& \operatorname{corr}\left(V T R_{i j}{ }^{t-1}, \Delta p_{i j}\right) \\
& =\frac{\operatorname{cov}\left(V T R_{i j}{ }^{t-1}, \Delta p_{i j}\right)}{d p\left(V T R_{i j}{ }^{t-1}\right) * d p\left(\Delta p_{i j}\right)}
\end{aligned}
$$

(7) Coeficiente de correlação entre a $V T R_{i j}$ final e a variação da participação do país j no total de patentes mundiais na subclasse $\mathrm{i}$.

$$
\begin{aligned}
& \operatorname{corr}\left(V T R_{i j}{ }^{t}, \Delta p_{i j}\right) \\
& =\frac{\operatorname{cov}\left(V T R_{i j}{ }^{t}, \Delta p_{i j}\right)}{d p\left(V T{R_{i j}}^{t}\right) * d p\left(\Delta p_{i j}\right)}
\end{aligned}
$$

$(\dagger)$ Sendo que: $p_{i j}=\frac{P_{i j}}{\sum_{j} P_{i j}}$; o sobrescrito $t-1$ refere-se ao período inicial; o sobrescrito $t$ refere-se ao período final; $\Delta$ indica variação; $c o v$ indica covariância; e $d p$ indica desvio padrão.

Fonte: elaboração própria com base em Urraca-Ruiz (2008) e Feitosa (2016).

O indicador de vantagem tecnológica revelada (VTR) é utilizado para mensurar a especialização tecnológica de determinado país $j$ em um dado campo tecnológico $i$. Através do VTR é possível detectar "fortalezas tecnológicas", ou seja, os campos tecnológicos onde a quota de patentes de um país é superior à quota mundial - isto é, evidenciando os campos tecnológicos nos quais o país apresenta vantagem tecnológica relativa.

Na representação algébrica do VTR, o subscrito $j$ indica o país, enquanto o subscrito $i$ indica a subclasse do IPC Green Inventory. Ainda: $P_{i j} P_{i j}$ é o número de patentes do país $j$ na subclasse $i ; \sum_{i} P_{i j}$ corresponde ao total de patentes do país $j$ em energias renováveis; $\sum_{j} P_{i j}$ compreende a soma das patentes mundiais na subclasse $i ; \sum_{i} \sum_{j} P_{i j}$ representa o total de patentes mundiais em ER. Um $V T R_{i j}$ superior a 1 indica que a participação das patentes da subclasse $i$ no total de patentes do país $j$ é superior à participação das patentes da subclasse $i$ no total de patentes mundiais, indicando a especialização do país $j$ na subclasse $i$. A taxa de variação da vantagem tecnológica revelada relacionada à variação da $V T R_{i j}$ entre os períodos é o indicador que capta as mudanças nas vantagens tecnológicas relativas ao longo do tempo. 
O indicador de nível de oportunidade tecnológica (OT) é utilizado para identificar os campos tecnológicos em que há maior apropriabilidade tecnológica. Isso porque pode-se supor que um maior nível de patenteamento indica uma maior possibilidade de exploração da área tecnológica. Aqui, tanto $\sum_{j} P_{i j}$ quanto $\sum_{i} \sum_{j} P_{i j}$ apresentam o mesmo significado exposto anteriormente. A variação entre os dois períodos representa o grau de dinamicidade da oportunidade tecnológica e está atrelado à existência de janelas de oportunidade (Urraca-Ruiz, 2008; Feitosa, 2016).

A dinâmica de especialização e mobilidade dos países entre dois períodos pode ser captada pelo índice de persistência (IP), apresentado na Equação (4). Onde, $C_{i}$ corresponde ao número de subclasses nas quais o país era especializado no período inicial, $C_{f}$ compreende o número de subclasses nas quais o país era especializado no período final e $C_{i f}$ representa o número de subclasses em que o país manteve a especialização nos dois períodos. Sendo que: $C_{t}=C_{i}+C_{i f}+C_{f}$. O índice IP varia entre 0 e 1 . Considerando que o índice de mobilidade é IM $=1-$ IP, tem-se que quando o IP assume valor 1, indica que a especialização se manteve a mesma nos dois períodos e, portanto, a persistência é máxima. Quando o IP assume valor zero, o IM será igual a 1, significando que o país abandonou totalmente o padrão de especialização do período inicial partindo para uma especialização totalmente diferente no período final, em cujo caso a mobilidade será máxima (Urraca-Ruiz, 2008).

A compreensão dos padrões de persistência e mobilidade (encontrados através do índice IP) pode ser feita pelo cálculo do coeficiente de correlação entre a $V T R_{i j}$ inicial e a variação da participação do país $j$ no total de patentes mundiais na subclasse $i$, bem como pelo cálculo do coeficiente de correlação entre a $V T R_{i j}$ final e a variação da participação do país $j$ no total de patentes mundiais na subclasse $i$. De acordo com Urraca-Ruiz (2008), quando o primeiro assume valor positivo, indica que há determinismo na direção da mudança tecnológica, ou seja, há persistência e cumulatividade, e quando o segundo é positivo, indica que a trajetória da atividade inovadora determina o padrão de especialização.

\section{III.2.1. Resultados}

Considerando o indicador VTR dos países no grupo das subclasses de ER, pode-se notar, entre o primeiro e o segundo períodos, um crescimento do número de situações de especializações dos países (com VTR >1) nessas tecnologias: de 96 para 136 (Quadro 3). Pode-se inferir que houve para esses países um maior esforço de patenteamento nesse grupo de subclasses, mas não de forma homogênea. Há países com maior número de situações de especializações tecnológicas - tais como 
China, Alemanha, Itália e Rússia - quando comparados com Nigéria, Indonésia e Irã, com poucas situações de especializações. Com as exceções de Japão e Coreia do Sul, todos os países apresentaram uma variação positiva no número de VTR $>1$, com destaque para a Turquia.

Quadro 3. Países: Número de Subclasses de Patentes em Energias Renováveis com VTR $>1$

\begin{tabular}{|c|c|c|c|c|}
\hline País & $\begin{array}{c}\text { Número } \\
\text { de VTR > } \\
1 \text { Primeiro } \\
\text { Período (A) }\end{array}$ & $\begin{array}{l}\text { Número de VTR } \\
>1 \text { Segundo } \\
\text { Período }(\mathrm{B})\end{array}$ & Soma $(A+B)$ & $\begin{array}{c}\text { Variação } \\
\text { (B-A)/ } \\
(\mathrm{A}) \\
(\%)\end{array}$ \\
\hline Alemanha & 7 & 10 & 17 & 43 \\
\hline Arábia Saudita & 0 & 0 & 0 & 0 \\
\hline Austrália & 7 & 9 & 16 & 29 \\
\hline Brasil & 6 & 8 & 14 & 33 \\
\hline Canadá & 5 & 10 & 15 & 100 \\
\hline China & 8 & 9 & 17 & 13 \\
\hline Coreia do Sul & 9 & 7 & 16 & -22 \\
\hline Espanha & 5 & 6 & 11 & 20 \\
\hline Estados Unidos & 2 & 4 & 6 & 100 \\
\hline França & 6 & 8 & 14 & 33 \\
\hline Índia & 4 & 8 & 12 & 100 \\
\hline Indonésia & 1 & 4 & 5 & 300 \\
\hline Irã & 1 & 4 & 5 & 300 \\
\hline Itália & 7 & 10 & 17 & 43 \\
\hline Japão & 9 & 2 & 11 & -78 \\
\hline México & 4 & 7 & 11 & 75 \\
\hline Nigéria & 1 & 1 & 2 & 0 \\
\hline Reino Unido & 5 & 10 & 15 & 100 \\
\hline Rússia & 9 & 9 & 17 & 100 \\
\hline Turquia & 2 & 10 & 12 & 400 \\
\hline Total & 98 & 136 & 232 & 42 \\
\hline
\end{tabular}

Fonte: elaboração própria. 
É válido ressaltar que um VTR superior a 1 não indica necessariamente que o país seja tecnologicamente superior naquela subclasse em relação aos demais, mas sim que o percentual de participação da referida subclasse nas patentes do país é superior à participação da subclasse nas patentes totais do grupo de países. Storti (2020) ilustra isso com o caso dos EUA; apesar de terem a maior participação em 12 das 13 subclasses de patentes no primeiro período, apresentaram VTR $>1$ em apenas duas delas (Biocombustíveis e OTEC). O mesmo ocorreu no segundo período, no qual tinham liderança em 9 das 13 subclasses, mas apresentaram VTR $>1$ em apenas 4 subclasses. $O$ caso do Japão é similar. Apesar desse país ter uma participação importante na grande maioria das subclasses, inclusive elevando a sua participação de $12.3 \%$ para $26.9 \%$ nas patentes entre os períodos (Quadro 5), registrou uma queda do número de subclasses com VTR $>1$, de 9 para 2 (em Energia solar e em Células de combustível).

Os casos dos EUA e do Japão mostram que o menor número de subclasses com VTR $>1$ comparativamente a outros países - como, por exemplo, o Brasil (que obteve um VTR maior que a unidade em 6 subclasses no primeiro período e em 8 , no segundo) - não significa que esses países tenham desvantagens estruturais, mas sim que apresentam especialização mais forte em certas áreas tecnológicas mais específicas.

O nível de oportunidade tecnológica de cada uma das subclasses de áreas tecnológicas nos dois períodos analisados e seus respectivos graus de dinamicidade são apresentados no Quadro 4.

Quadro 4. Nível de oportunidade tecnológica e grau de dinamicidade da oportunidade tecnológica das subclasses tecnológicas

\begin{tabular}{|c|c|c|c|c|}
\hline \multirow{2}{*}{ Sigla } & \multirow{2}{*}{ Subclasse tecnológica } & \multicolumn{2}{|c|}{$\begin{array}{c}\text { Nível de } \\
\text { oportunidade } \\
\text { tecnológica }\end{array}$} & \multirow{2}{*}{$\begin{array}{c}\text { Grau de } \\
\text { dinamicidade } \\
\text { da } \\
\text { oportunidade } \\
\text { tecnológica }\end{array}$} \\
\hline & & $\begin{array}{c}1^{\mathrm{o}} \\
\text { período }\end{array}$ & $\begin{array}{c}2^{\circ} \\
\text { período }\end{array}$ & \\
\hline BioC & Biocombustíveis & 0.576 & 0.384 & $-33.38 \%$ \\
\hline IGCC & $\begin{array}{c}\text { Ciclo combinado de } \\
\text { gaseificação integrada (IGCC) }\end{array}$ & 0.006 & 0.008 & $27.32 \%$ \\
\hline $\mathrm{CC}$ & Células de combustível & 0.126 & 0.172 & $36.72 \%$ \\
\hline
\end{tabular}




\begin{tabular}{|c|c|c|c|c|}
\hline PGS & $\begin{array}{c}\text { Pirólise ou gaseificação de } \\
\text { biomassa }\end{array}$ & 0.011 & 0.014 & $29.07 \%$ \\
\hline WTE & $\begin{array}{c}\text { Energia de resíduos de atividades } \\
\text { humanas - Waste-to-Energy }\end{array}$ & 0.083 & 0.071 & $-13.98 \%$ \\
\hline $\mathrm{EH}$ & Energia hidráulica & 0.012 & 0.024 & $93.30 \%$ \\
\hline OTEC & $\begin{array}{l}\text { Conversão da energia térmica } \\
\text { dos oceanos (OTEC) }\end{array}$ & 0.0002 & 0.0004 & $110.27 \%$ \\
\hline $\mathrm{EO}$ & Energia eólica & 0.022 & 0.023 & $3.37 \%$ \\
\hline ES & Energia solar & 0.115 & 0.237 & $105.76 \%$ \\
\hline EG & Energia geotérmica & 0.021 & 0.029 & $36.36 \%$ \\
\hline OTUC & $\begin{array}{l}\text { Outros tipos de produção ou } \\
\text { utilização de calor }\end{array}$ & 0.004 & 0.006 & $68.98 \%$ \\
\hline $\mathrm{CR}$ & Utilização de calor residual & 0.023 & 0.031 & $33.96 \%$ \\
\hline PEMEN & $\begin{array}{l}\text { Produção de energia mecânica } \\
\text { a partir de energia muscular }\end{array}$ & 0.0001 & 0.0004 & $176.54 \%$ \\
\hline
\end{tabular}

Fonte: elaboração própria.

Como pode ser observado, em ambos os períodos, a subclasse bicombustiveis aparece como detentora do maior nível de oportunidade tecnológica, isto é, a que apresentou maior percentual de patenteamento dentro dos registros totais do grupo de ER. Destacam-se também as subclasses energia solar, células de combustível e, um pouco mais atrás, o WTE. As subclasses detentoras dos menores níveis de oportunidade tecnológica são aquelas cujo desenvolvimento de tecnologias ainda se encontra em fase incipiente de desenvolvimento e difusão, como são os casos da OTEC e da produção de energia mecânica a partir de energia muscular. Considerando as tecnológicas mais avançadas nos graus de dinamicidade da oportunidade tecnológica, a energia solar e a célula de combustível se destacam nos seus graus de dinamicidade, indicando que são subclasses que apresentam janelas de oportunidades. Biocombustiveis e WTE apresentaram graus negativos, demonstrando estagnação. 
Nesse aspecto, pode-se argumentar que as subclasses com melhores resultados são as que estão pautadas por trajetórias tecnológicas e campos científicos já consolidados e/ou que se beneficiam de fatores de cumulatividade do conhecimento. Adicionalmente - e para além de questões referentes à base do conhecimento - deve-se lembrar a importância dos aspectos sociais e institucionais relacionados às condições de oportunidade tecnológica (Malerba, 2002). Assim, áreas tecnológicas de biocombustíveis, energia solar células de combustivel, e de WTE contam também com vetores positivos e mais desenvolvidos relacionados às instituições reguladoras, aos valores sociais, aos incentivos e às estruturas de mercado relativamente às demais áreas. Esses aspectos ajudam à ocorrência da coevolução sinérgica entre a base de conhecimento, as tecnologias e as instituições, que se autorreforçam por meio de processos de path-dependence, solidificando as suas trajetórias tecnológicas e elevando, concomitantemente, as suas oportunidades tecnológicas.

No que diz respeito à participação relativa dos países no número de patentes de ER (Quadro 5), verifica-se o destaque tecnológico principalmente dos EUA, Alemanha, Reino Unido e França. No primeiro período, os EUA eram responsáveis por cerca de $48 \%$ de todos os registros de patentes no grupo de ER. No segundo período, a participação relativa caiu para $30.25 \%$. As participações da Alemanha, Reino Unido e França também caíram, notadamente em decorrência do aumento do nível de patenteamento dos países asiáticos, tais como Japão, China, e Coreia do Sul. O Japão passou de uma participação de $12.32 \%$ para $26.93 \%$, aproximando-se dos Estados Unidos.

Quadro 5. Participação dos países no número de patentes de energias renováveis

\begin{tabular}{|c|c|c|c|c|c|}
\hline \multirow[t]{2}{*}{ País } & \multicolumn{2}{|c|}{$\begin{array}{c}\text { Participação dos países nas } \\
\text { patentes } \\
(\%)\end{array}$} & \multicolumn{2}{|c|}{$\begin{array}{c}\text { Coeficiente de } \\
\text { correlação entre a } \\
\text { variação na participação } \\
\text { total de patentes e o }\end{array}$} & \multirow[t]{2}{*}{$\begin{array}{c}\text { Índice de } \\
\text { persistência }\end{array}$} \\
\hline & $1^{\circ}$ período & $2^{\circ}$ período & $\begin{array}{l}\text { VTR } \\
\text { inicial }\end{array}$ & VTR final & \\
\hline Alemanha & 13.32 & 11.21 & 0.23 & 0.3 & 0.29 \\
\hline Arábia Saudita & 0 & 0 & - & - & - \\
\hline Austrália & 0.03 & 0.22 & 0.07 & 0.05 & 0.27 \\
\hline Brasil & 0.21 & 0.49 & -0.07 & -0.06 & 0.28 \\
\hline
\end{tabular}




\begin{tabular}{|c|c|c|c|c|c|}
\hline Canadá & 3.72 & 2.79 & -0.49 & 0.17 & 0.21 \\
\hline China & 1.13 & 6.56 & -.08 & 0 & .26 \\
\hline Coreia do Sul & 1.61 & 6.51 & 0 & 0.42 & 0.30 \\
\hline Espanha & 0.84 & 1.29 & 0.03 & 0.08 & 0.21 \\
\hline Estados unidos & 48.02 & 30.25 & -0.28 & -0.33 & 0.25 \\
\hline França & 5.28 & 4.87 & -0.10 & 0.02 & 0.22 \\
\hline Índia & 0.50 & 0.87 & -0.05 & -0.07 & 0.20 \\
\hline Indonésia & 0 & 0.03 & -0.93 & -0.04 & 0 \\
\hline Irã & 0 & 0.01 & -0.88 & 0.06 & 0.17 \\
\hline Itália & 1.54 & 1.7 & 0.12 & 0.41 & 0.3 \\
\hline Japão & 12.32 & 26.93 & 0.34 & 0.41 & 0.15 \\
\hline México & 0.13 & 0.16 & -0.24 & -0.02 & 0.27 \\
\hline Nigéria & 0 & 0 & -0.74 & 0.74 & 0 \\
\hline Reino unido & 7.84 & 3.94 & -0.06 & 0.11 & 0.21 \\
\hline Rússia & 0.92 & 0.67 & 0.16 & 0.19 & 0.29 \\
\hline Turquia & 0.03 & 0.22 & -0.33 & 0.05 & 0.14 \\
\hline
\end{tabular}

Fonte: elaboração própria.

Nessa perspectiva, e considerando o mesmo período temporal, o trabalho de Storti (2020) mostra adicionalmente que:

a) considerando os dados desagregados da participação relativa dos países no total das patentes mundiais nas 13 subclasses de ER durante os dois períodos, os Estados Unidos se destacam num leque mais amplo das diversas tecnologias. No primeiro período, o país destacava-se como líder tecnológico em doze das treze 
subclasses, sendo a única exceção a subclasse PEMEN, liderada esta pela Coreia do Sul. A Alemanha postava-se como segundo país mais relevante em nove subclasses (Biocombustíveis, IGCC, Pirólise ou gaseificação de biomassa, Aproveitamento de energia a partir de residuos de atividades humanas, Energia hidráulica, Energia eólica, Energia solar, Energia geotérmica e Utilização de calor residual); o Japão liderava nas outras três subclasses. No segundo período, os Estados Unidos perderam a liderança tecnológica em três subclasses, sendo ultrapassados pelo Japão nas subclasses Células de combustivel e Energia solar, e pela Alemanha na subclasse Utilização de calor residual. A Coreia do Sul também perdeu a liderança na subclasse PEMEN para a China. Este país também desbancou o Japão da posição na subclasse Outros tipos de produção ou utilização de calor;

b) em relação à participação relativa das subclasses no total de patentes do grupo de ER em cada país, no primeiro período, Biocombustíveis era a subclasse mais expressiva nas patentes de todos os países. Essa subclasse representava mais da metade das patentes registradas em ER em doze dos países. Cerca de $64.28 \%$ das patentes estadunidenses registradas em ER eram referentes a Biocombustiveis; na Índia esse percentual era de $84.26 \%$; no Brasil, de $66.67 \%$. Mesmo nos países que apresentavam uma distribuição mais equilibrada de patentes, tais como Japão, Alemanha e Coreia do Sul, o percentual de patentes em Biocombustiveis foi elevado, com participações de $40.29 \%$, 47.27\%, e 48.94\%, respectivamente nesses países.

No segundo período, o quadro mudou um pouco, configurando-se numa maior dispersão das patentes. A subclasse Biocombustíveis perdeu participação relativa no total de patentes de cada um dos países, deixando de ser a subclasse mais representativa em quatro deles. Na Coreia do Sul, o setor de Células de combustivel assumiu a principal posição com $27.95 \%$ de todas as patentes do país em ER. Nos casos do Japão, Itália e Turquia, o setor de Energia solar foi a subclasse mais importante, com uma participação de $29.55 \%, 29.19 \%$, e $24.32 \%$, respectivamente nesses países. A segunda subclasse mais importante nas patentes japonesas foi Células de combustivel, enquanto a de Energia solar foi a segunda mais importante nas patentes sul-coreanas, e a de Energia hidráulica foi nas patentes turcas. A subclasse Energia solar ganhou participação nas patentes em quase todos os países entre o primeiro e o segundo períodos; a exceção foi a Turquia, em decorrência do aumento da diversificação nas áreas de patenteamento.

A dinâmica de especialização tecnológica (ou não) dos países em ER entre dois períodos pode ser captada pelo indice de persistência (IP). Quando o IP assume valor 1, indica que a especialização se manteve a mesma nos dois períodos e, portanto, a persistência é máxima. Quando o IP assume valor zero, significa 
que o país abandonou totalmente o padrão de especialização do período inicial, caracterizando alta mobilidade. Pelo Quadro 5, observa-se que o IP médio dos países no período foi de apenas 0.24 , indicando assim alta mobilidade no patenteamento dos países. Ou seja, os países apresentaram mudanças significativas em seus padrões de especialização tecnológica em ER de um período para o outro. O país com o maior IP foi a Coreia do Sul, mas, mesmo assim, apresentou um índice de apenas 0.30 . No outro lado do espectro, encontra-se a Turquia com o menor IP (0.14), seguida pelo Japão com 0.15. Estados Unidos e China tiveram resultados próximos à média geral, 0.25 e 0.26 respectivamente. O Brasil apresentou um IP acima da média (0.28). A característica de baixa persistência foi ratificada pelos resultados do coeficiente de correlação entre a variação na participação no total de patentes do país e o VTR inicial, que se mostrou positivo para apenas seis países (Japão, Alemanha, Rússia, Itália, Austrália e Espanha, em ordem decrescente). Isso significa que a especialização tecnológica de apenas seis países da amostra era influenciada por fatores de cumulatividade.

O Japão, apesar de ter apresentado o segundo menor índice de persistência, apresentou o mais elevado coeficiente de correlação entre o VTR inicial e a variação na participação no total de patentes do país, indicando presença de cumulatividade. Além disso, ao analisar o coeficiente de correlação entre o VTR final e a variação na sua participação no total de patentes (que quando positivo indica que a trajetória da própria atividade inovadora determina o padrão de especialização), verifica-se um coeficiente positivo e superior ao coeficiente de correlação entre o VTR inicial e a variação na participação total de patentes do país. Isso significa que o padrão de especialização tecnológica japonês se guiou tanto por fatores de cumulatividade, bem como por fatores relacionados à própria atividade inovadora, mas estes últimos se sobressaíram em relação aos primeiros.

O ocorrido com o Japão se repete para os demais cinco países que apresentaram indícios de influência de fatores de cumulatividade na determinação de trajetórias tecnológicas. Os países que não apresentaram coeficiente de correlação entre o VTR final e a variação na participação no total de patentes do país positivo foram México, Indonésia, Brasil, Índia e Estados Unidos. Para esses países, portanto, não foi possível determinar quais fatores guiaram seus padrões de especialização tecnológica. 


\section{III.3. Análise de decomposição estrutural via método shift-share}

O método de componentes de variação (shift-share) - ou análise de decomposição estrutural - decompõe o crescimento (ou a variação) de uma variável em fatores distintos que influenciam o comportamento da variável entre dois períodos. Esse método é utilizado em diversos estudos empíricos, dentre os quais a dinâmica tecnológica. Neste artigo, o método shift-share é aplicado para analisar como a variação da participação de um país no total de patentes mundiais está relacionada a três efeitos: o efeito quota tecnológica, o efeito tecnológico estrutural, e o efeito tecnológico de adaptação (Laursen, 1999; Urraca-Ruiz, 2008; Feitosa, 2016).

O efeito quota tecnológica permite verificar a parcela da variação na participação do país no total de patentes mundiais relacionada ao dinamismo geral de sua atividade patenteadora, assumindo que o peso de cada subclasse se mantenha constante entre os dois períodos. Ou seja, verifica-se o crescimento (ou decrescimento) da quota de patentes ao se considerar a estrutura idêntica em ambos os períodos.

O efeito tecnológico estrutural permite verificar a parcela da variação na participação do país no total de patentes mundiais que se deve ao dinamismo (ou estagnação) das subclasses nas quais o país se encontrava especializado no período inicial. Assim, verifica-se o crescimento (ou decrescimento) da quota de patentes originado pela "adequada" (ou "inadequada") estrutura de especialização tecnológica do país.

O efeito tecnológico de adaptação permite verificar a parcela da variação na participação do país no total de patentes mundiais decorrente de seus movimentos de entrada e saída de subclasses dinâmicas e estagnadas. Para avaliar se o efeito de adaptação é de entrada nas subclasses "corretas" (dinâmicas) ou de saída das subclasses "erradas" (estagnadas), é necessária sua decomposição em dois outros efeitos: o efeito adaptação de crescimento tecnológico e o efeito adaptação de estagnação tecnológica. De modo que o primeiro mensura a parcela de crescimento (ou decrescimento) da quota tecnológica advinda da entrada (ou saída) do país em subclasses dinâmicas. O segundo quantifica a parcela de crescimento (ou decrescimento) da quota tecnológica decorrente da saída (ou entrada) do país de subclasses estagnadas.

Assim, a taxa de variação da participação de determinado país sobre o total de patentes mundiais $\Delta p_{j}$ pode ser decomposta em três efeitos principais (Equação 8): (i) efeito quota tecnológica; (ii) efeito tecnológico estrutural; (iii) efeito tecnológico de adaptação. 


$$
\Delta p_{j}=\sum_{i}\left(\Delta p_{i j} o_{i}^{t-1}\right)+\sum_{i}\left(p_{i j}^{t-1} \Delta o_{i}\right)+\sum_{i}\left[\Delta p_{i j} \Delta o_{i}\right]
$$

(i)

(ii)

(iii)

Onde $p_{j}$ representa a participação do país $j$ no total de patentes mundiais, Equação (9); $p_{i j}$ representa a participação do país $j$ no total de patentes mundiais na subclasse $i$, Equação (10); e $o_{i}$ compreende a participação da subclasse $i$ no total de patentes mundiais, Equação (11); $t-1$ indica período inicial e $t$, período final; e $\Delta$ simboliza variação entre os dois períodos.

$$
\begin{gathered}
p_{j}=\frac{\sum_{i} P_{i j}}{\sum_{i} \sum_{j} P_{i j}} \\
p_{i j}=\frac{P_{i j}}{\sum_{j} P_{i j}} \\
o_{i}=\frac{\sum_{j} P_{i j}}{\sum_{i} \sum_{j} P_{i j}}
\end{gathered}
$$

Tem-se que o efeito tecnológico de adaptação (iii) pode ser dividido da seguinte forma:

$$
\sum_{i}\left[\Delta p_{i j} \Delta o_{i}\right]=\sum_{i}\left[\Delta p_{i j} \frac{\left(\Delta o_{i}+\left|\Delta o_{i}\right|\right)}{2}\right]+\sum_{i}\left[\Delta p_{i j} \frac{\left(\Delta o_{i}-\left|\Delta o_{i}\right|\right)}{2}\right]
$$

Portanto, os efeitos de forma completa são representados por:

$$
\begin{gathered}
\Delta p_{j}=\sum_{i}\left(\Delta p_{i j} o_{i}^{t-1}\right)+\sum_{i}\left(p_{i j}^{t-1} \Delta o_{i}\right)+\sum_{i}\left[\Delta p_{i j} \frac{\left(\Delta o_{i}+\left|\Delta o_{i}\right|\right)}{2}\right]+ \\
\sum_{i}\left[\Delta p_{i j} \frac{\left(\Delta o_{i}-\left|\Delta o_{i}\right|\right)}{2}\right]
\end{gathered}
$$

A Equação (13) corresponde aos quatro efeitos definidos: (i) efeito quota tecnológica; (ii) efeito tecnológico estrutural; (iii) efeito adaptação do crescimento tecnológico; (iv) efeito adaptação da estagnação tecnológica. Na medida em que o crescimento de patentes pode ser usado como medida da oportunidade tecnológica, os três últimos fatores representam uma medida do acesso de um dado país a áreas de ER com um grau relativamente elevado de oportunidade tecnológica. 
Se o efeito tecnológico estrutural de um país for positivo e alto, isso significa que o país estava especializado "corretamente" no período inicial (áreas em ER que experimentaram dinamicidade na oportunidade tecnológica). Isso pode ser também uma medida de "persistência" em sentido positivo ou negativo. Se os dois últimos efeitos são elevados e positivos, significa que o país se moveu para setores com oportunidade tecnológica dinâmica ou saiu de áreas que registraram oportunidade tecnológica estagnada (Urraca-Ruiz, 2008; Feitosa, 2016).

O Quadro 6 mostra os resultados da análise shift-share dos países com a decomposição dos diferentes efeitos e a variação da participação dos países no total de patentes entre os períodos.

Quadro 6. Resultados da análise shift-share: decomposição dos diferentes efeitos e a variação da participação dos países no total das patentes entre os períodos

\begin{tabular}{|c|c|c|c|c|c|}
\hline \multirow{2}{*}{ País } & \multirow{2}{*}{$\begin{array}{c}\text { Efeito } \\
\text { quota } \\
\text { tecnológica }\end{array}$} & \multirow{2}{*}{$\begin{array}{c}\text { Efeito } \\
\text { tecnológico } \\
\text { estrutural }\end{array}$} & \multicolumn{2}{|c|}{ Efeito tecnológico de adaptação } & \multirow{2}{*}{$\begin{array}{c}\text { Variação da } \\
\text { participação } \\
\text { do país no } \\
\text { total de } \\
\text { patentes }\end{array}$} \\
\hline & & & $\begin{array}{c}\text { Efeito } \\
\text { crescimento } \\
\text { tecnológico }\end{array}$ & $\begin{array}{c}\text { Efeito } \\
\text { estagnação } \\
\text { tecnológica }\end{array}$ & \\
\hline Japão & 0.1178 & 0.0173 & 0.0322 & -0.0212 & 0.1461 \\
\hline China & 0.0558 & 0.0006 & 0.0106 & -0.0123 & 0.0547 \\
\hline Coreia do Sul & 0.0426 & 0.0009 & 0.0119 & -0.0065 & 0.0490 \\
\hline Espanha & 0.0045 & -0.0003 & 0.0011 & -0.0007 & 0.0046 \\
\hline Índia & 0.0052 & -0.001 & 0.0007 & -0.0012 & 0.0037 \\
\hline Brasil & 0.0037 & -0.0001 & 0.0002 & -0.0009 & 0.0028 \\
\hline Turquia & 0.0014 & 0.0001 & 0.0004 & -0.0001 & 0.0018 \\
\hline Itália & 0.0003 & 0.0014 & -0.0001 & 0.0000 & 0.0015 \\
\hline Indonésia & 0.0003 & -0.0000 & 0.0000 & -0.0001 & 0.0003 \\
\hline
\end{tabular}




\begin{tabular}{|c|c|c|c|c|c|}
\hline México & 0.0004 & -0.0001 & 0.0000 & -0.0001 & 0.0003 \\
\hline Irã & 0.0001 & -0.0000 & 0.0000 & -0.0000 & 0.0001 \\
\hline Arábia Saudita & 0.0000 & 0.0000 & 0.0000 & 0.0000 & 0.0000 \\
\hline Nigéria & -0.0000 & -0.0000 & 0.0000 & 0.0000 & -0.0000 \\
\hline Rússia & -0.0025 & 0.0013 & -0.0014 & 0.0000 & -0.0025 \\
\hline França & -0.0031 & -0.0020 & -0.0003 & 0.0013 & -0.0041 \\
\hline Canadá & -0.0061 & -0.0005 & -0.0026 & -0.0002 & -0.0093 \\
\hline Austrália & -0.0107 & 0.0017 & -0.0038 & 0.00156 & -0.0112 \\
\hline Alemanha & -0.0318 & 0.0158 & -0.0118 & 0.0067 & -0.0211 \\
\hline Reino Unido & -0.0377 & -0.0054 & -0.0054 & 0.0095 & -0.0390 \\
\hline Estados Unidos & -0.1400 & -0.0297 & -0.0321 & 0.0240 & -0.1778 \\
\hline
\end{tabular}

Fonte: elaboração própria

Os dados mostram a liderança do Japão, da China e da Coreia do Sul, tanto na variação positiva na participação das patentes totais, bem como no efeito quota tecnológica, demonstrando terem sido os países com maior dinamismo na atividade geral patenteadora em ER. O trabalho de Storti (2020) destaca o importante desempenho da China no período, em que esse país galgou sete posições em Energia hidráulica, seis posições em Biocombustiveis, Pirólise ou gaseificação de biomassa, Energia eólica e Energia solar, e cinco posições em Células de combustível e Aproveitamento de energia a partir de resíduos de atividades humanas.

Os países que apresentaram perda de participação nas patentes totais foram: Nigéria, Rússia, França, Canadá, Austrália, Alemanha, Reino Unido e Estados Unidos. Em todos esses países, o efeito quota tecnológica se mostrou negativo e mais expressivo que os demais efeitos, o que indica queda no dinamismo geral da atividade patenteadora do país. Deve-se alertar, novamente, que a perda de participação de um país pode estar relacionada ao aumento da atividade patenteadora dos demais, e não propriamente a um declínio da sua atividade patenteadora. Os Estados Unidos exemplificam esse aspecto, uma vez que, embora o país tenha sido 
aquele que registrou a maior variação negativa da participação no total de patentes, como visto anteriormente, permaneceu na liderança da maioria das subclasses ao longo do segundo período.

Entretanto, nem todos os casos se assemelham ao estadunidense. O Canadá e o Reino Unido, por exemplo, chegaram a registrar queda no número absoluto de patentes em Células de combustivel e Biocombustiveis, respectivamente. No ranking de países mais participativos em termos de patentes de ER, o Canadá foi ultrapassado em um total de oito subclasses das treze, enquanto o Reino Unido perdeu posições em doze subclasses (mesmo número da Rússia). Mesmo a competitiva Alemanha perdeu posições em um total de seis subclasses (mesmo número da França), apresentando ganho de posição apenas em Utilização de calor residual. O caso mais expressivo é o da Austrália que perdeu posição em todos os setores, tendo ocorrido a queda mais acentuada na subclasse Energia eólica, na qual o país perdeu 7 posições (Storti, 2020).

Os países que apresentaram efeito tecnológico estrutural negativo e, portanto, apresentaram uma especialização "inadequada" no período inicial foram todos países nos quais a subclasse estagnada Biocombustiveis representava mais do que $50 \%$ das patentes registradas pelo país (Espanha, Índia, Brasil, Indonésia, México, Irã, Nigéria, França, Canadá, Reino Unido e Estados Unidos). Nos demais países, esse efeito se mostrou positivo, com destaque para Japão e Alemanha.

Os países que apresentaram efeito adaptação de crescimento tecnológico positivo, em ordem decrescente de amplitude, foram Japão, Coreia do Sul, China, Espanha, Índia, Turquia, Brasil, México, Indonésia, Irã e Nigéria. Para todos esses países, a combinação dos ganhos registrados em cada subclasse dinâmica com seus respectivos graus de dinamicidade foi de maior amplitude que as perdas registradas pelo país em subclasses dinâmicas. No outro lado do espectro, os países que apresentaram efeito adaptação de crescimento tecnológico negativo, em ordem decrescente de amplitude, foram Estados Unidos, Alemanha, Reino Unido, Austrália, Canadá, Rússia, França e Itália.

No que diz respeito ao efeito tecnológico de adaptação, um fato que se evidencia é que os países que apresentaram efeito adaptação de crescimento tecnológico positivo apresentaram efeito adaptação de estagnação tecnológico negativos, e vice-versa. Como o primeiro efeito mensura o aumento ou diminuição de participação em setores dinâmicos e o segundo mensura o aumento ou diminuição de participação em setores estagnados, evidencia-se que os países que aumentaram sua participação relativa no total de patentes mundiais fizeram isso não apenas em 
setores dinâmicos, mas também em setores estagnados. E os países que perderam participação no total de patentes internacionais o fizeram tanto em setores dinâmicos quanto em setores estagnados. Em síntese, o resultado confirma o que outros indicadores já indiciavam, ou seja, os países que cresceram competitivamente na área de ER apresentaram crescimento geral em todas as subclasses, da mesma forma que os países que perderam espaço na área perderam espaço nas subclasses em geral. A exceção foi o Canadá que apresentou ambos os efeitos negativos, indicando aumento de participação em setores estagnados e diminuição de participação em setores dinâmicos.

Somando os dois efeitos, o resultado representa o efeito tecnológico de adaptação. Quando positivo, esse efeito indica - considerando os aumentos e diminuições de participação nos setores dinâmicos e estagnados - que o país foi capaz de focar em áreas tecnológicas dinâmicas em relação às estagnadas; ou seja, transformou o seu padrão inovativo no sentido de se especializar em setores dinâmicos e se "desespecializar" em setores estagnados. Os países que tiveram efeito tecnológico de adaptação positivo foram Japão, Coreia do Sul, Espanha, Turquia, Nigéria, França, e Reino Unido.

\section{CONCLUSÕES}

Em decorrência dos possíveis limites do esgotamento das reservas fósseis, as recorrentes crises mundiais no setor petrolífero e as crescentes externalidades negativas socioambientais derivadas do excesso de utilização de energias fósseis, em diversos países há esforços tecnológicos para a produção e o uso crescente de ER. Delineia-se um processo de transição para uma matriz energética baseada em fontes renováveis que é cada vez mais desejada socialmente. No entanto, o processo de transição não é neutro para agentes econômicos (empresas e consumidores), setores produtivos, e para as economias, inclusive sob o aspecto da competição geoeconômica entre países.

Nesse sentido, compreender os caminhos trilhados pelos países mais representativos no consumo de energia no mundo, no que diz respeito às suas inovações ligadas ao campo das ER, fornece indícios do rumo e das configurações do próprio processo de impactos e de mudança do paradigma energético. Por essa razão, o objetivo do presente estudo foi verificar por meio de alguns indicadores selecionados de patentes - e pela realização de uma análise shift-share - os esforços científicos e tecnológicos realizados por esses países. 
Os resultados demonstraram que áreas como Conversão da energia térmica dos oceanos e Dispositivo para produção de energia mecânica a partir de energia muscular estão em fases iniciais de desenvolvimento e, portanto, ainda não apresentam trajetórias tecnológicas fortemente consolidadas. O campo de Biocombustíveis foi o mais importante e com o maior número de patenteamento, embora tenha apresentado redução no percentual de participação em todos os países, em função do crescimento das demais subclasses de patentes, chegando inclusive a perder a posição de liderança no patenteamento em países como Japão e Coreia do Sul. A área de Energia solar foi a que apresentou a trajetória inovativa mais dinâmica. A subclasse não apenas assumiu a segunda posição em termos de nível de oportunidade, como foi uma das que mais se destacou em termos de grau de oportunidade, fato que revela o acelerado crescimento dessa área tecnológica.

Os Estados Unidos estiveram à frente no patenteamento para a maioria das subclasses. Países europeus como Reino Unido e Alemanha, que tradicionalmente se destacam em políticas de incentivo ao uso de ER revelaram, no entanto, perda de participação internacional e queda no ranking em número de patentes em ER. No sentido oposto, os países asiáticos - notadamente. Japão. Coreia do Sul e China - apresentaram forte dinâmica patenteadora, elevaram não apenas a quantidade absoluta, como também ganharam espaço relativo no cenário internacional. O Japão assumiu a liderança inovativa no setor que mais se destacou no período: a Energia solar. Esse país ascendeu no ranking internacional em oito subclasses, a Coreia do Sul em onze, e a China em todas as treze. A ascensão chinesa foi a mais expressiva, galgando sete posições em Energia hidráulica e seis posições em Biocombustiveis e Pirólise ou gaseificação de biomassa. Os grandes produtores de petróleo, incluindo países desenvolvidos como o Canadá, demonstraram, de forma geral, fraca atividade patenteadora.

A análise shift-share indicou a existência de uma certa estratégia de esforços de inovação em fontes energéticas renováveis mais geral e abrangente. Os países que apresentaram crescimento em determinada subclasse apresentaram crescimento em todas as subclasses de patentes. De modo oposto, os países que perderam espaço em uma subclasse perderam espaço de forma geral. Isso indica que os esforços inovativos realizados e a construção de mecanismos e/ou organizações institucionais em prol do desenvolvimento de ER estão ligados à forma em que o país enfrenta a questão energética como um todo, e não a uma determinada subclasse em específico. Vale dizer, os países, em vez de focarem em uma ou em poucas fontes energéticas alternativas "eleitas" como substitutas da fonte de combustíveis fósseis, contemplam um leque mais amplo de tecnologias em energias renováveis. 


\section{REFERÊNCIAS}

Albino, V., Ardito, L., Dangekico, R. M., \& Petruzelli, A. M. (2014). Understanding the development trends of low-carbon energy technologies: A patent analysis. Applied Energy, 13 (C), 836-854. doi: 10.1016/j.apenergy.2014.08.012 Archibugi D., \& Pianta M. (1994). Innovation surveys and patents as technology indicators: the state of the art. Journal of Evolutionary Economics, 4(1), 17-33. doi: https://doi.org/10.1007/BF01200835

Bayer, P., Dolan, L, \& Urpelainen, J. (2013). Global patterns of renewable energy innovation, 1990-2009. Energy for Sustainable Development, 17(3), 288295. doi: https://doi.org/10.1016/j.esd.2013.02.003

Bueno, C. S., Silveira, J. M. F. J., Buainain, A. M., \& Dal Poz, M. E. S. (2018). Applying an IPC network to identify the bioenergy technological frontier. Revista Brasileira de Inovação, 17(2), 259-286. doi: http://dx.doi. org/10.20396/rbi.v17i2.8652020

Bonnet, C.,Hache, E.,Seck, G. S., Simoën, M.,\& Carcanague, S. (2019). Who's winning the low-carbon innovation race? An assessment of countries' leadership in renewable energy Technologies. International Economics, 160 (C), 31-42. doi: 10.1016/j.inteco.2019.07.006

Cefis, H. (2003). Is there persistence in innovative activities? International Journal of Industrial Organization, 21(4), 489-515.

Criekemans, D. (2018). The Geopolitics of Renewables. In D. Scholten. Geopolitics of Renewable Energy Game and its Potential Impact upon Global Power Relations (pp. 37-73). Germany: Springer.

Faria, L. (2020). Revolução energética em curso - Custo médio ponderado de geração de eletricidade a partir de fontes renováveis (dados do IRENA - International Renewable Energy Agency). Disponível em https://valoradicionado. wordpress.com/2020/08/05/revolucao-energetica-em-curso/

Feitosa, P. H. A. (2016). Estrutura tecnológica e mudanças climáticas no Brasil: um estudo exploratório a partir de estatísticas de patentes. Revista Brasileira de Inovação, 15(1), 61-86

Frankfurt School-UNEP Centre/BNEF (2020), Global Trends in Renewable Energy Investment, 2020. Disponível em http://www.fs-unep-centre.org. Acessado em: 03/03/2021

Fu, W., Li, C., Ondrich, J., \& Popp, D. (2018). Technological spillover effects of state renewable energy policy: evidence from patent counts. (NBER, Working paper No. 25390).

International Renewable Energy Agency-IRENA (s. f.). Dados disponíveis em: https://www.irena.org/statistics 
Laursen, K. (1999). The impact of technological opportunity on the dynamics of trade performance. Structural Change and Economic Dynamics, 10(3), 341357.

Malerba, F., \& Orsenigo, L. (1993). L'accumulazione delle capacita'tecnologiche nell'industria italiana (1969-1984). In C. Filippini. Innovazione tecnologica e servizi alle imprese. Milan: Franco Angeli.

Malerba, F., (2002). Sectoral systems of innovation and production. Research policy, 31(2), 247-264.

Renewable Energy Policy Network for the 21st Century-REN21 (2019). Renewables 2019 Global Status Report. Disponível em http://repository.usp. ac.fj/11648/1/gsr_2019_full_report_en.pdf

Samant, S., Pooja, T., \& Hatfeld, D. E. (2020). Does the focus of renewable energy policy impact the nature of innovation? Evidence from emerging economies. Energy Policy, 137. doi: https://doi.org/10.1016/j.enpol.2019.111119

Soete, L. (1987). The impact of technological innovation on international trade patterns: the evidence reconsidered. Research Policy, 16(2-4), 101-130.

Storti, D. R. F. (2020). Tecnologias em energias renováveis: uma análise de alguns indicadores de patentes para países selecionados no período 1992-2018. Santa Maria: UFSM.

Urraca-Ruiz, A. (2008). Persistência versus mudança estrutural da especialização tecnológica do Brasil. Economia e Sociedade, 17(3), 403-427.

World Intellectual Property Organization -WIPO. (2019). IPC Green Inventory. Disponível em https://www.wipo.int/classifications/ipc/green-inventory/ home

Yuehong, L., Khan, Z., Alvarez-Alvarado, M., Zhang, Y., Huang, Z., \& Imran, M. (2020). A Critical Review of Sustainable Energy Policies for the Promotion of Renewable Energy Sources. Sustainability, 12(12), 5078. doi:10.3390/ su12125078

(C) 2022 por los autores; licencia no exclusiva otorgada a la revista Estudios económicos. Este artículo es de acceso abierto y distribuido bajo los términos y condiciones de una licencia Atribución-No Comercial 4.0 Internacional (CC BY-NC 4.0) de Creative Commons. Para ver una copia de esta licencia, visite http://creativecommons.org/licenses/by-nc/4.0 
